\title{
Huella de carbono de equipos térmicos convencionales bajo la perspectiva de la ingeniería de procesos
}

\author{
Alejandro Garza Galicia \\ a.garza@univa.mx \\ Universidad del Valle de Atemajac, Campus Guadalajara. \\ Guadalajara - México \\ Isaura de los Ángeles Valenzuela Patlán \\ isaura.vp@hotmail.com \\ Universidad del Valle de Atemajac, Campus Guadalajara. \\ Guadalajara - México \\ Belkis Sulbarán-Rangel \\ Belkis.sulbaran@academicos.udg.mx \\ Universidad de Guadalajara. Centro Universitario de Tonalá. \\ Tonalá - México
}

\section{RESUMEN}

La presente investigación se basa en la comparación de la huella de carbón debido a las emisiones que se desprenden hacia la atmósfera de tres máquinas térmicas, una caldera de $25 \mathrm{CC}$ a gas LP, un motor de combustión interna de un transporte de carga utilizando combustible diésel y un calentador de gas, las tres máquinas operando a condiciones de operación en tiempo real. La determinación de la huella de carbón se hizo teóricamente mediante el uso de factores de emisión que proponen dos metodologías reportadas y se compara con el método experimental acorde a la ingeniería del proceso, es decir, tomado en cuenta las condiciones mecánicas, térmicas y los transitorios que se presenten a través de un monitoreo con analizadores de gases de combustión. Se han desarrollado diferentes metodologías para el cálculo de la huella de carbono y cada una de ellas arroja un resultado diferente, por lo que se presenta un análisis de las metodologías y el planteamiento de la determinación de la huella de carbono desde el punto de vista de la Ingeniería de procesos, obteniéndose una variación de la huella de carbón de hasta un $74.5 \%$ mayor que con las metodologías establecidas.

Palabras clave: cambio climático; combustión; emisiones; proceso transitorio termodinámico. 


\title{
Carbon footprint of conventional thermal equipment from the perspective of process engineering
}

\begin{abstract}
The present research is based on quantification analysis of the carbon footprint due to the emissions that are released to the atmosphere by three thermal machines, a $25 \mathrm{CC}$ boiler with LP gas, an internal combustion engine of a cargo transport using diesel fuel and a gas heater, all three machines at real-time operating conditions. The carbon footprint was made theoretically by using emission factors that are proposed by two reported methodologies and it is compared with the experimental method according to the process engineering; that is, taking into account the mechanical, thermal transitory conditions that are presented through combustion gas analyzers monitoring. Different methodologies have been developed for calculating the carbon footprint and each one shows a different result, in this sense, an analysis of the methodologies and the carbon footprint calculation is presented from the point of view of Process Engineering obtaining a variation in the carbon footprint of up to $74.5 \%$ greater than with the established methodologies.
\end{abstract}

Keywords: climate change; combustion; emissions; thermodynamic transitory process.

Artículo recibido: 20. Julio. 2021

Aceptado para publicación: 18. Agosto. 2021

Correspondencia: Belkis.sulbaran@academicos.udg.mx

Conflictos de Interés: Ninguna que declarar 


\section{INTRODUCCIÓN}

El incremento continuo de las necesidades energéticas de la población humana es actualmente una de las principales problemáticas ambientales, puesto que gran parte de la energía se produce a partir de combustible fósiles, generando una gran cantidad de gases de efecto invernadero (GEI); acorde a las autoridades ambientales nacionales, en 2015 se liberaron 685 millones de toneladas de huella de carbono $\mathrm{CO}_{2}$ equivalente, deteriorando la biodiversidad y contribuyendo al cambio climático (INECC, 2018; Zhou et al., 2019).

La humanidad está enfrentando uno de los problemas más severos de su historia en materia ambiental; la emisión de diferentes gases contaminantes a la atmósfera ha provocado impacto en todos los ámbitos, sin duda que sus efectos van desde los macrosistemas como el derretimiento en los polos hasta los escases del agua y el incremento de las enfermedades (Andrews \& Smirnov, 2020). Por tanto, la medición en la temperatura global de la tierra y el registro de las emisiones que la provocan se han vuelto tópicos vitales para organismos internacionales, nacionales y científicos (Hao, Liu, \& Michaels, 2020). A partir de la cuantificación de las emisiones de gases de efecto invernadero generadas por procesos, se calcula la huella de carbono para indicar el grado de impacto que tienen las emisiones contaminantes sobre el medio ambiente y la salud humana (Beeftink, Hofs, Kramer, Odegard, \& van der Wal, 2021; Ottelin, Heinonen, \& Junnila, 2018). La huella de carbono representa la cantidad de emisiones netas de gases de efecto invernadero, expresadas en bióxido de carbono $\mathrm{CO}_{2}$ equivalente, es decir de todos aquellos gases que generan efecto invernadero, que derivan de actividades de producción o consumo de bienes y servicios ya sea a nivel residencial o industrial (Fenner et al., 2018). Este indicador muestra el grado de impacto que se está produciendo en el medio ambiente, en los seres vivos y sobre todo el potencial daño a la salud y el equilibrio de los procesos naturales. Mientras más elevado sea este indicador mayor será el efecto nocivo, cada vez es más común su cuantificación a través de los inventarios de emisiones, sin embargo, no han sido suficientes las acciones para disminuirla y al contrario cada vez se incrementa (Beeftink et al., 2021; Mancini et al., 2016).

Espíndola y Valderrama (2012) mencionan que existen cuatro métodos principales para calcular de la Huella de Carbono en empresas, organizaciones, servicios, procesos y productos. Éstos analizan el ciclo de vida del producto para la obtención de datos, desde 
el origen de las materias primas necesarias hasta su adecuada disposición final. Cada método usa un enfoque diferente, dependiendo de hasta dónde las emisiones de otros procesos influyen en el proceso en estudio de la empresa (Espíndola \& Valderrama, 2012). Los métodos mencionados estiman la huella de carbono sumando las variables que inciden en la producción de gases de efecto invernadero mediante algún modelo matemático. Se ha identificado que estas cuatro metodologías reportadas por Espíndola y Valderrama, 2012, denotan un factor de emisión fijo para los productos y los servicios. Por otra parte otros autores reporta una metodología para tres diferentes gases en un esquema específico para el área de telecomunicaciones (Radonjič \& Tompa, 2018), para biomateriales (Pang, Pun, Chow, \& Ishak, 2014), para la industria del cemento (Cagiao, Gómez, Doménech, Mainar, \& Lanza, 2011) y para emisión para combustibles (INECC, 2018). De esta forma se identifica que existen diferentes metodologías y planteamientos para determinar el factor de emisión y el cálculo de la huella de carbono, de aquí la importancia de seleccionar la adecuada, y acorde a las condiciones de la construcción, instalación, fabricación, método o proceso. De acuerdo con esto se ha observado que el principio de los cálculos presenta una falta de correlación con los procesos reales, en el caso de procesos de combustión se abre una serie de condiciones relativos a los transitorios termoquímicos que dependen de los sistemas mecánicos. Por ejemplo las condiciones de los anillos de los pistones en un motor de cuatro tiempos permiten la infiltración del aceite del cárter a las cámaras de combustión por lo que no solo se quema la gasolina (Weise, 1990), al igual que el desprendimiento de partículas de metal en la cámara afectan la química de la combustión (Kohan, 2000), en el caso de las máquinas térmicas, como la caldera la aparición de transitorios termoquímicos por la inestabilidad de los flujos de combustible cuando se prende y se apaga el quemador, al igual que se presenta otro transitorio en la transferencia de calor de la cámara de combustión a los tubos donde circula el agua(Barbosa, 2018; Delgadillo, 2012). De aquí se desprende una vertiente muy importante en la determinación de la huella de carbón desde un punto de vista del uso de los factores y el utilizar los principios de la ingeniería de procesos al medir y monitorear las maquinas bajo las condiciones de operación. Por tanto, el objetivo de esta investigación es la cuantificación de la huella de carbón debido a las emisiones que se desprenden hacia la atmósfera de tres máquinas térmicas, una caldera de $25 \mathrm{CC}$ a gas LP, un motor de combustión interna de un transporte de carga utilizando combustible 
diésel y un calentador de gas casero, con la finalidad de hacer la determinación de las tres máquinas operando a condiciones de operación real y tomando en cuenta los transitorios propios de cada equipo.

\section{MATERIALES Y MÉTODOS}

Los equipos térmicos utilizados en esta investigación fueron: una caldera de $25 \mathrm{CC}$ a gas LP (Marca Mass Ter-Cal, Modelo: LC-1800-EE, México) en uso continuo, un motor de combustión interna de un transporte de carga utilizando combustible diésel (Marca Cummins, modelo Diesel 6.7, Estados Unidos de America) en pleno tráfico y un calentador de gas (Marca Optimus, modelo; OR-10, México) con usuarios demandando intermitentemente el agua caliente. La medición de los gases se realizó con un analizador para CO2 marca TES modelo 1370 NDIR, para CO y una bomba para la mancha de Hollín. Con este equipo se midió la temperatura y el flujo de gases.

Para determinar la huella de carbón con la ingeniería del proceso, se toma al equipo como una operación unitaria sometido a las condiciones de carga y demanda, a la perdida de calor por transferencia con las condiciones de arranque y alcance del estado estable a nivel termodinámico(Çengel \& Boles, 2015; Delgadillo, 2012). Para probar la propuesta se determinó la huella de carbono de los equipos térmicos mencionados bajo las condiciones referidas en la Tabla 1.

Tabla 1. Parámetros de operación máquinas térmicas

\begin{tabular}{|c|c|c|c|c|c|c|c|c|}
\hline Equipo & Combustible & $\begin{array}{c}\text { Consumo } \\
\text { Kg }\end{array}$ & $\begin{array}{c}\text { Tiempo } \\
\text { horas }\end{array}$ & $\begin{array}{c}\mathbf{V} \\
\mathbf{m} / \mathbf{s}\end{array}$ & $\begin{array}{c}\text { Diámetro } \\
\text { descarga } \\
\text { in }\end{array}$ & $\begin{array}{c}\text { Temperatura } \\
\text { Gases Equipo } \\
{ }^{\circ} \mathbf{C}\end{array}$ & $\begin{array}{c}\text { Temperatura } \\
\text { Ambiente }{ }^{\circ} \mathbf{C}\end{array}$ & Opacidad \\
\hline $\begin{array}{c}\text { Caldera } 25 \\
\text { CC }\end{array}$ & Gas LP & 3.9 & 2.2 & 0.85 & 12 & 77 & 28 & 0.2 \\
\hline $\begin{array}{c}\text { Motor } \\
\text { diesel }\end{array}$ & Diesel & 14.9 & 2.7 & 2.9 & 2 & 62 & 33 & 4 \\
\hline $\begin{array}{c}\text { Calentador } \\
\text { de agua }\end{array}$ & Gas LP & 0.06 & 0.28 & 0.97 & 4 & 92 & 29 & 0.2 \\
\hline
\end{tabular}

A partir del consumo de combustible se calculó teóricamente la huella de carbono en $\mathrm{Kg}$, como se indica en la ecuación (1).

Huella de carbón $\left(\mathrm{Kg} \mathrm{CO}_{2}\right)=(\mathrm{Kg}$ combustible $) *$ (Factor de emisión $)$

Es decir, multiplicando los kilogramos de cada combustible por el factor de conversión correspondiente de las metodologías reportadas por Instituto Nacional de Ecología y Cambio Climático (INECC, 2018) que serán comparadas con las emisiones en términos de la producción de $\mathrm{CO}_{2}$ equivalente. Se seleccionaron condiciones de operación en un 
día de trabajo normal en los cuales están presentes fenómenos transitorios, es decir las demandas sobre los equipos estaban determinados por las condiciones de las instalaciones o del tráfico en el caso del motor diesel.

\section{RESULTADOS Y DISCUSIÓN}

Uno de los aspectos relevantes en la operación, fue tomar a estas máquinas térmicas, como un sistema termodinámico en donde las interacciones de calor y trabajo responden a las demandas, esto es la operación unitaria que demanda calor en el caso de la caldera y el calentador de agua, y trabajo en la flecha para el motor diésel(Çengel \& Boles, 2015; Wardach, Palka, Paplicki, Prajzendanc, \& Zarebski, 2020). Se considera de suma importancia ya que calcula teóricamente la huella en los equipos de proceso bajo una condición de estado estable y como se vio en los equipos se presentan frecuentemente transitorios de temperatura del agua en la caldera y el calentador mientras que en el motor la demanda de potencia (Geankoplis, 2003).

Para determinar la huella de carbón experimental se calculó a partir de la concentración promedio obtenida, considerando el caudal y el tiempo para obtener la masa total de cada uno de los contaminantes registrados. Como el monóxido de carbono CO no se considera gas de efecto invernadero por lo que no se contabiliza en la huella de carbón solo se considera el $\mathrm{CO}_{2}$ (Ghosh, 2020). El registro de los promedios de lectura y cálculo de la huella equivalente en kilogramos se presenta en la Tabla 2.

Tabla 2. Calculo de huella de carbón experimental

\begin{tabular}{|l|c|c|c|c|c|c|c|}
\hline \multicolumn{1}{|c|}{ Equipo } & $\begin{array}{c}\text { Caudal } \\
\mathbf{m}^{\mathbf{3}} \mathbf{s}\end{array}$ & $\begin{array}{c}\text { tiempo } \\
\mathbf{s}\end{array}$ & $\begin{array}{c}\mathbf{C O} \\
\mathbf{p p m}\end{array}$ & $\begin{array}{c}\mathbf{C O}_{2} \\
\mathbf{p p m}\end{array}$ & $\begin{array}{c}\mathbf{C O} \\
\mathbf{k g}\end{array}$ & $\begin{array}{c}\mathbf{C O}_{2} \\
\mathbf{k g}\end{array}$ & $\begin{array}{c}\mathbf{C O}_{2 \text { equivalente }} \\
\mathbf{k g}\end{array}$ \\
\hline $\begin{array}{l}\text { Caldera } 25 \\
\text { CC }\end{array}$ & 0.0365 & 7920.0 & 31.5 & 60.8 & 9.1 & 17.57 & 17.57 \\
\hline Motor & 0.0059 & 9720.0 & 4313.6 & 3262.6 & 246.4 & 186.4 & 186.40 \\
\hline $\begin{array}{l}\text { Calentador } \\
\text { casero }\end{array}$ & 0.0079 & 1008.0 & 33.6 & 50.7 & 0.3 & 0.40 & 0.40 \\
\hline
\end{tabular}

\section{1 ppm parte por millón $=0.001$ grms/litro}

La huella de carbono, a partir de un combustible se toman en cuenta principalmente las emisiones a partir del consumo y tipo que se quema; este cálculo se basa en una reacción química estequiometricas donde se obtiene $\mathrm{CO}_{2}$ y agua a partir de la oxidación de un combustible, como se muestra en la ecuación (2), la gasolina $\mathrm{C}_{8} \mathrm{H}_{18}$ puede reemplazarse por el combustible al que se esté refiriendo el análisis. 
$\mathrm{C}_{8} \mathrm{H}_{18}+\mathrm{O}_{2} \quad \mathrm{CO}_{2}+\mathrm{H}_{2} \mathrm{O} \longrightarrow$ (2)

Al balancear la reacción acorde a (Wallace \& Hobbs, 2006) se obtiene la ecuación (3) en donde se observa que el producto de emisión contaminante tiene un volumen significativamente mayor a la cantidad de combustible que se utiliza.

$\mathrm{C}_{8} \mathrm{H}_{18}+12.5 \mathrm{O}_{2} \quad 8 \mathrm{CO}_{2}+9 \mathrm{H}_{2} \mathrm{O} \longrightarrow(3)$

Como se ve el oxígeno oxida completamente al hidrocarburo, sin embargo, los fenómenos transitorios, la eficiencia de la combustión y la posible infiltración del aceite del carter hace producir una combustión incompleta, refiriéndose al uso de gasolina, por lo que se obtendrá también monóxido de carbono (CO) y óxidos de nitrógeno (NOX), éstos últimos siendo 298 veces más contaminante como gas de efecto invernadero que el $\mathrm{CO}_{2}$ (Espíndola \& Valderrama, 2012). En el caso de la combustión incompleta de productos como el diesel, se obtendrían como productos $\mathrm{CO}$, óxido de nitrógeno (NOX), óxidos de azufre (SOX) y partículas de hidrocarburos (PPM HC). La huella de carbono en este caso no considera al CO, sin embargo, desde el punto de vista de la combustión, la reacción química que presenta $\mathrm{CO}$ produce otros gases de mayor efecto como los NOX e hidrocarburos no quemados (HC), que en el caso del motor diésel se ve reflejado en la mancha de hollín en una escala muy alta ya que en este caso el vehículo es modelo 1956 y presenta físicamente humo negro y en parte de la operación humo blanco que demuestra que pasa aceite del cuerpo del motor a las cámaras de combustión. Esto quiere decir que hay los factores de emisión no toman en cuenta condiciones mecánicas o del aceite que se usa en los automotores.

Para el cálculo de la huella de carbón a partir de los factores de emisión, se establece la relación a partir del consumo de combustible, dependiendo del tipo de combustible es el factor. Los factores de emisión (INECC, 2018) se indican por combustible y equipo, el cálculo con cada factor se reporta en la Tabla 3.

Tabla 3. Calculo de huella de carbón con factores INECC, 2014

\begin{tabular}{|l|c|c|c|c|c|}
\hline Equipo & Consumo & Factor & Factor & $\mathbf{C O}_{2}$ & $\mathbf{C O}_{2}$ \\
\hline
\end{tabular}




\begin{tabular}{|l|c|c|c|c|c|}
\hline & & $\begin{array}{c}\mathbf{C O}_{2} \\
\text { INECC } \\
\mathbf{k g} / \mathbf{k g C o m b}\end{array}$ & $\begin{array}{c}\mathbf{C O}_{\mathbf{2}} \\
\mathbf{I P C C} \\
\mathbf{K g} / \mathbf{k g C o m b}\end{array}$ & $\begin{array}{c}\text { INECC } \\
\mathbf{k g}\end{array}$ & $\begin{array}{c}\text { IPCC } \\
\mathbf{~ k g}\end{array}$ \\
\hline $\begin{array}{l}\text { Caldera 25 } \\
\text { CC }\end{array}$ & 3.9 & 3.0 & 2.9085 & 11.7 & 11.34 \\
\hline Motor & 14.9 & 3.129 & 3.18 & 46.62 & 47.38 \\
\hline $\begin{array}{l}\text { Calentador } \\
\text { casero }\end{array}$ & 0.06 & 3 & 2.9085 & 0.18 & 0.177 \\
\hline
\end{tabular}

Como se puede observar en la Tabla 3, se presenta una diferencia menor del 3\% entre metodología del INECC y la del reporte del panel intergubernamental de cambio climático (IPCC), esto debido al valor del factor que se reporta, sin embargo, son factores que solo toman en cuenta las características del combustible.

$\mathrm{Al}$ analizar la máquina como operación unitaria considerando las interacciones de flujo, la demanda de energía, las pérdidas de calor, las condiciones mecánicas de los elementos mecánicos, el tiempo de vida, es decir, las condiciones reales a las que se ve sometido, se observa una diferencia sustancial con los valores obtenidos con los factores de emisión de las metodologías, como se ilustra en la Tabla 4.

\section{Tabla 4. Análisis comparativo de huella de carbón}

\begin{tabular}{|c|c|c|c|c|c|}
\hline \multirow{2}{*}{ Equipo } & \multirow{2}{*}{$\begin{array}{c}\mathrm{CO}_{2} \\
\text { Medido } \\
\text { kg }\end{array}$} & \multirow{2}{*}{$\begin{array}{c}\text { Gradiente } \\
\text { INECC } \\
\text { Teórico } \\
\text { kg }\end{array}$} & \multirow{2}{*}{$\begin{array}{c}\text { Gradiente } \\
\text { IPCC } \\
\text { Teórico } \\
\text { kg }\end{array}$} & \multicolumn{2}{|c|}{$\begin{array}{l}\text { Diferencia entre el valor } \\
\text { teórico y el medido }\end{array}$} \\
\hline & & & & $\begin{array}{c}\% \\
\text { INECC }\end{array}$ & $\begin{array}{c}\% \\
\text { IPCC }\end{array}$ \\
\hline $\begin{array}{l}\text { Caldera } 25 \\
\text { CC }\end{array}$ & 17.57 & 5.86 & 6.22 & 33.40 & 35.43 \\
\hline Motor diesel & 186.40 & 139.78 & 139.02 & 74.99 & 74.58 \\
\hline $\begin{array}{l}\text { Calentador } \\
\text { casero }\end{array}$ & 0.40 & 0.22 & 0.23 & 54.64 & 56.02 \\
\hline
\end{tabular}

En la Tabla 4 se observa que la medición directa de acuerdo con el reporte del panel intergubernamental de cambio climático (\%IPCC) es mayor en todos los casos; existe una diferencia sustancial de al menos $33.4 \%$ mayor en el caso de la caldera con respecto a la metodología del INECC, para el motor diésel de 74 \% mayor que en prácticamente las dos metodologías y para el calentador casero alrededor de $54 \%$, esto quiere decir que las condiciones de los equipos, la operación y la química de la combustión son determinantes en la producción de emisiones contaminantes y no solo del bióxido de carbono. 


\section{CONCLUSIÓN}

Los factores de emisión tienen diferentes puntos de referencia y cálculo, con lo cual la magnitud de la huella de carbón es diferente, de aquí la importancia de identificar y caracterizar el proceso y sus características de operación, sobre todo cuando se trata de equipos de transferencia de calor, masa y combustión, en donde se presentan fenómenos transitorios, condiciones de fricción, desprendimiento de materiales que afectan la química del proceso, estos efectos no son cuantificados ya que dependen del equipo y su condición de funcionamiento. En el análisis presentado se midió directamente las emisiones en la fuente caracterizando a la máquina como una operación unitaria, de esta forma se aisló el proceso del sistema y se identificó que los procesos están sometidos a fenómenos transitorios tanto físicos como químicos que marcaron el incremento de la huella de carbón.

La huella de carbón que se obtuvo bajo la operación real es mayor que la determinada con las metodologías tradicionales ya que se midió con analizadores directamente con las condiciones de operación. Bajo la condición de que las máquinas y equipos trabajan con procesos inestables se dejan de cuantificar desmesuradas cantidades de emisiones contaminantes a nivel global que representa información crítica acerca de la calidad del aire.

Al establecer una metodología basada en la reacción química del combustible y el balanceo molar se dejan de tomar en cuenta una gran cantidad de fenómenos asociados a la operación y la condición de los equipos, como se observó en los resultados, se pueden presentar diferencias de hasta el $74.99 \%$ en las máquinas analizadas, aunque hay propuestas de metodologías que toman en cuenta algunos factores, se requiere profundizar desde el punto de vista experimental, revisar las condiciones de los equipos, la edad, ya que como en el caso del motor diésel, hay más de 60 años de operación del motor. Estas perspectivas hacer suponer que, en un gran número de casos, las emisiones a la atmosfera por huella de carbón en equipos de proceso, podrían ser mayores que los reportados en inventarios y cálculo global de la huella de carbón, por lo que los impactos al medio ambiente y al calentamiento global seguirán incrementándose.

\section{LISTA DE REFERENCIAS}


Andrews, T. M., \& Smirnov, O. (2020). Who feels the impacts of climate change? Global $\begin{array}{lll}\text { Environmental Change, } & \text { 65, }\end{array}$ doi:https://doi.org/10.1016/j.gloenvcha.2020.102164

Barbosa, J. (2018). Termodinámica para ingenieros. México

Beeftink, M., Hofs, B., Kramer, O., Odegard, I., \& van der Wal, A. (2021). Carbon footprint of drinking water softening as determined by life cycle assessment. $\begin{array}{llll}\text { Journal of Cleaner } & \end{array}$ doi:https://doi.org/10.1016/j.jclepro.2020.123925

Cagiao, J., Gómez, B., Doménech, J. L., Mainar, S. G., \& Lanza, H. G. (2011). Calculation of the corporate carbon footprint of the cement industry by the application of MC3 methodology. Ecological Indicators, 11(6), 1526-1540. doi:https://doi.org/10.1016/j.ecolind.2011.02.013

Çengel, Y. A., \& Boles, M. A. (2015). Termodinámica (8a. ed.). USA.

Delgadillo, S. (2012). Termodinámica básica. . IPN México

Espíndola, C., \& Valderrama, J. O. (2012). Huella del Carbono. Parte 1: Conceptos, Métodos de Estimación y Complejidades Metodológicas. Información tecnológica, 23, 163-176.

Fenner, A. E., Kibert, C. J., Woo, J., Morque, S., Razkenari, M., Hakim, H., \& Lu, X. (2018). The carbon footprint of buildings: A review of methodologies and applications. Renewable and Sustainable Energy Reviews, 94, 1142-1152. doi:https://doi.org/10.1016/j.rser.2018.07.012

Geankoplis, C. J. (2003). Transport Processes and Separation Process Principles: (includes Unit Operations): Prentice Hall Professional Technical Reference.

Ghosh, A. (2020). Possibilities and Challenges for the Inclusion of the Electric Vehicle (EV) to Reduce the Carbon Footprint in the Transport Sector: A Review. Energies, 13(10), 2602.

Hao, F., Liu, X., \& Michaels, J. L. (2020). Social Capital, carbon dependency, and public response to climate change in 22 European countries. Environmental Science \& Policy, 114, 64-72. doi:https://doi.org/10.1016/j.envsci.2020.07.028

INECC. (2018). Inventario Nacional de Emisiones de Gases y Compuestos de Efecto Invernadero. $\quad$ Retrieved from https://www.gob.mx/inecc/acciones-y- 
programas/inventario-nacional-de-emisiones-de-gases-y-compuestos-de-efectoinvernadero

Kohan, A. L. (2000). Manual de calderas : principios operativos de mantenimiento, construcción, instalación, reparación, seguridad, requerimientos y normativas (v1). España: McGraw-Hill Interamericana.

Mancini, M. S., Galli, A., Niccolucci, V., Lin, D., Bastianoni, S., Wackernagel, M., \& Marchettini, N. (2016). Ecological Footprint: Refining the carbon Footprint calculation. Ecological Indicators, 61, 390-403. doi:https://doi.org/10.1016/j.ecolind.2015.09.040

Ottelin, J., Heinonen, J., \& Junnila, S. (2018). Carbon and material footprints of a welfare state: Why and how governments should enhance green investments. Environmental Science \& Policy, $\quad 86, \quad 1-10$. doi:https://doi.org/10.1016/i.envsci.2018.04.011

Pang, M. M., Pun, M.-Y., Chow, W.-S., \& Ishak, Z. A. M. (2014). Carbon footprint calculation for thermoformed starch-filled polypropylene biobased materials. Journal of Cleaner Production, 64, 602-608.

Radonjič, G., \& Tompa, S. (2018). Carbon footprint calculation in telecommunications companies - The importance and relevance of scope 3 greenhouse gases emissions. Renewable and Sustainable Energy Reviews, 98, 361-375. doi:https://doi.org/10.1016/j.rser.2018.09.018

Wallace, J., \& Hobbs, P. (2006). Atmospheric Science (Second Edition). Estados Unidos de America: Academic Press.

Wardach, M., Palka, R., Paplicki, P., Prajzendanc, P., \& Zarebski, T. (2020). Modern Hybrid Excited Electric Machines. Energies, 13(22), 5910.

Weise, J. H. (1990). Manual diesel de reparación y mantenimiento 1978-84: automóviles, camiones y tractores, motores y chasis. Barcelona: Océano Centrum.

Zhou, N., Zhang, J., Khanna, N., Fridley, D., Jiang, S., \& Liu, X. (2019). Intertwined impacts of water, energy development, and carbon emissions in China. Applied Energy, 238, 78-91. doi:https://doi.org/10.1016/j.apenergy.2018.12.085 